\title{
Estado trófico de la madre vieja Gota e' Leche, Yotoco, Valle del Cauca, Colombia. Basada en parámetros físicos, químicos y biológicos
}

\author{
Trophic state of the wetland "Gota e'Leche, Yotoco, Valle del Cauca state, \\ Colombia. Based on biological, physichal and chemical parameters
}

\author{
Díaz P, Juan ${ }^{1}$ Biol, Ortiz F, Guido ${ }^{1}$ Biol, Caicedo N, Rubiel ${ }^{1}$ Biol, Zambrano P, Leonidas ${ }^{*}$ M.Sc, \\ Zamora, Hilldier ${ }^{3}$ M.Sc, Naundorf, Gerardo ${ }^{3}$ M.Sc.
}

Universidad del Cauca, Facultad de Ciencias Naturales, Exactas y de la Educación, Departamento de Biología, ${ }^{1}$ Programa de Biología. ${ }^{2}$ Grupo de Estudios Ambientales (G.E.A). ${ }^{3}$ Grupo de Estudios Recursos Hidrobiológicos Continentales (GERHC), Cauca, Colombia.

\section{Keywords:}

Wetland; anthropic intervention; trophic state; eutrophic; hyper eutrophic; lacustrine.

\section{Palabras Clave:}

Humedal; madre vieja; antrópico; estado trófico; eutrófico; hipereutrófico; lacustre.

\section{Abstract}

One of the most representative wetlands Cauca Valley State is the one called "Gota e' Leche", located in the town of Yotoco. This ecosystem is important because it is the habitat of many species of animals and native plants. The region where is located is a tropical dry forest (Bs-T) (HOLDRIDGE, 1978). The wetland has had numerous trophic changes because the anthropogenic activities carry on the area (sugar mills, farmers, etc.), This study determined the trophic state of the wetland using biological parameters such as primary productivity, chlorophyll, riparian flora and aquatic macro invertebrates, as well as physical and chemical such as water temperature, salinity, dissolved gases and others. According to our data, we determined that the wetland has a eutrophic state prone to hyper eutrophication. The system is continuously subjected to disturbance by human activities that change the characteristics of the ecosystem. As an indicator of this change, it is noticeable that in some sectors the marshy vegetation shows succession process belonging to a transition between aquatic and terrestrial ecosystems. For this reason, it is important the get more and meaningful knowledge of their successional stage in order to implement actions that will enable their restoration and conservation.

\section{Resumen}

Los humedales más representativos dentro del departamento del Valle del Cauca son las madre vieja, una de ellas es Gota e' Leche, ubicada en el municipio de Yotoco, este sistema es importante porque es el hábitat de muchas especies de animales y plantas nativas del Departamento; perteneciente a la zona de vida: bosque seco tropical (Bs-T) (HOLDRIDGE, 1978). El humedal presenta una serie de alteraciones tróficas causadas por actividades de origen antrópico (agricultura, ganadería, etc.) que recaen sobre los ecosistemas acuáticos de la Región. Para conocer el estado trófico se determinaron parámetros biológicos como la productividad primara, clorofila, flora acuática y ripariana, macro invertebrados acuáticos, al igual que parámetros físicos y químicos; se realizaron mediciones de temperatura, salinidad, gases disueltos y otros asociados a la condición del sistema. Al comparar los datos basados en los parámetros anteriores, se determinó que el humedal presenta un comportamiento eutrófico con tendencia a la hiper eutroficación; esto se debe a que el sistema continuamente está sometido a perturbaciones antrópicas y como indicador de este cambio se nota en algunos sectores vegetación de tipo palustre, que dentro del proceso sucesional es la transición entre los ecosistemas acuáticos y terrestres. Por esta razón, es importante el pleno conocimiento de su estado sucesional, para con base en ello planear e implementar las acciones que posibiliten su restauración y conservación. 


\section{Introducción}

Los humedales son las áreas inundadas o saturadas por aguas superficiales (dulce o salada) o subterráneas y con flora y fauna adaptadas a tales condiciones. Entre las definiciones de humedales más aceptadas está la utilizada por la convención RAMSAR (1971) (Artículo 1), que los define como: "Extensiones de marismas, pantanos, turberas, o aguas de régimen natural o artificial, permanentes o temporales, estancadas o corrientes, dulces, salobres o saladas, incluyendo las extensiones de aguas marinas cuya profundidad en marea baja no exceda los seis metros".

En Colombia la disposición constante o temporal de agua a lo largo de todo el año, es una situación que favorece el desarrollo exitoso de una amplia diversidad de flora, fauna y microorganismos que interactúan en complejas relaciones para mantener un equilibrio ecológico de alta fragilidad. Factores como régimen climático, complejidad orogénica y ubicación biogeográfica se han combinado de forma excepcional para permitir que el recurso hídrico sea abundante y modele el paisaje tropical formando ríos, estuarios, pantanos, ciénagas y lagunas, entre otros (GUERRERO, 1998).

Los procesos de afectación humana en los humedales, no son independientes de la dinámica natural de estos sistemas (CARPENTER y NOTTINGHAM, 1998). Esta debe verse como una perturbación que actúa sobre la dinámica natural del sistema, y cuyo efecto depende de la magnitud, intensidad y tasa de recurrencia de la misma (aspectos externos), como también del estado del sistema y de su capacidad de retornar al estado de pre-perturbación o resiliencia (aspectos internos). En este sentido, los conflictos entre las actividades humanas y la conservación o uso sustentable de humedales se presentan en varios órdenes de magnitud, jerárquicamente organizados (WAYNE y SÉLLER, 1984).

La historia de cambios negativos de los humedales colombianos se ha debido directa o indirectamente a los patrones de distribución de los asentamientos humanos en el país. El cambio de una economía basada en la agricultura extensiva y el pastoreo de ganado vacuno a un sistema dominado por la agricultura de monocultivo y a la concentración de las poblaciones urbanas durante las últimas décadas, ha sido responsable por la completa destrucción de vastos sistemas acuáticos tales como los del altiplano Cundiboyacense y el Valle del Cauca (GUERRERO, 1998).

Dentro del valle geográfico del río Cauca y particularmente en el departamento del Valle del Cauca, se encuentran sistemas lénticos los cuales presentan una alimentación hídrica ya sea directa o por nivel freático con el río Cauca, conocidas como madre viejas o paleocauses. Las madres viejas como los demás humedales son sistemas complejos con una ecología muy frágil; el desconocimiento de la importancia de estos ecosistemas ha originado su degradación e incluso su destrucción. Se encuentran amenazados por una serie de problemas como son las represas, las canalizaciones, la contaminación y el avance de las fronteras agropecuarias (GUERRERO, 1998) que en el departamento del Valle del Cauca es bien conocido por la implantación del monocultivo de la caña de azúcar (Saccharum officinarum $\mathrm{L}$ ).

La preocupación inicial es la determinación del estado trófico basado en una caracterización ecológica en la madre vieja Gota e' Leche o Román en el municipio de Yotoco, donde la frontera agrícola ha causado la perdida casi total de la vegetación típica de esta zona de vida que según Holdridge corresponde a bosque seco tropical (bs-T). Para tal estudio se tuvieron en cuenta parámetros físicos, químicos y biológicos, los cuales revelan el estado ecológico de este humedal.

El presente estudio muestra el estado sucesional de la de la madre vieja Gota e' Leche de Yotoco, Valle del Cauca, basada en parámetros físicos, químicos y biológicos, para con base en ello planear e implementar las acciones que posibiliten su restauración y conservación.

\section{Materiales y métodos}

Entre los meses de febrero y septiembre de 2006, se realizaron jornadas de campo para llevar a cabo la caracterización ecológica de la madre vieja Gota e' Leche, teniendo en cuenta parámetros físicos, químicos y biológicos como la cuantificación de clorofila a, macroinvertebrados acuáticos epicontinentales MAE, vegetación acuática (macrófitas) y vegetación riparia.

Zona de estudio: Se encuentra ubicada dentro del sistema de humedales del valle geográfico del río Cauca en el departamento del Valle del Cauca, flanco oriental de la cordillera Occidental, municipio de Yotoco, su posición geográfica es: $03^{\circ} 47^{\prime} 386^{\prime \prime} \mathrm{N}$ y $76^{\circ} 23^{\prime}$ 654 " W, a una altura sobre el nivel del mar de $985 \mathrm{~m}$. El área aproximada del humedal es de 11,1 hectáreas (Figura 1).

Inicialmente se realizaron algunas caracterizaciones batimétricas como la longitud, anchura y profundidad mediante el empleo de decámetros comerciales.

La vegetación ribereña se encuentra dispuesta en remanentes de árboles y plantas típicas de esta zona de vida que correspondiente a bosque seco tropical (bs-T) 

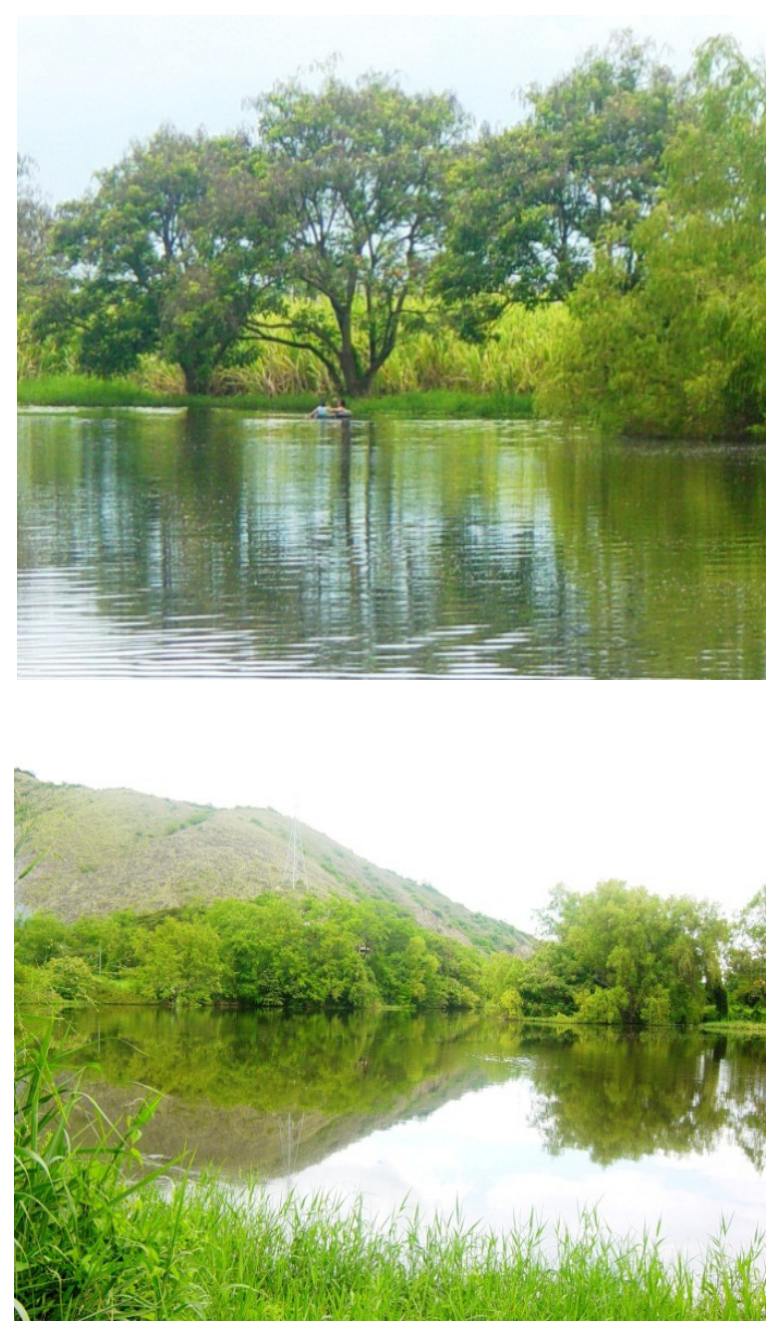

Figura 1. Rasgos generales de la zona de estudio. A) Madre vieja rodeada vegetación ribereña y B) Árboles y de caña de azúcar. Fuente: Zambrano (2010).

(HOLDRIDGE, 1978). La actividad antrópica que influencia directamente sobre el humedal es el monocultivo de caña de azúcar (Saccharum officinarum $L$ ). Se observa un canal de irrigación que aporta aguas al humedal en tiempos de alta pluviosidad. No se observa la franja de protección que debe tener cada humedal. Se observó adicionalmente el uso de las aguas para la ganadería vacuna y pesca artesanal realizada por los moradores la zona y turistas.

Muestreos: Los muestreos se realizaron en la zona litoral entre las regiones de vegetación emergente y flotante, y otra localizada en la zona central del humedal o zona limnética de acuerdo con los métodos establecidos por la EPA para la caracterización de ríos y humedales (EPA, 1999).
Proceso metodológico: A continuación, se describen los procesos metodológicos desarrollados en la zona de estudio del humedal.

Determinación de características físico-químicas hídricas. La determinación de los parámetros físicos y químicos hídricos se adelantó con base en los métodos estándar (APHA, 1999). Para tales efectos se consideraron: Temperatura hídrica $\left(\mathrm{T}^{\circ}\right)$ usando un teletermómetro YSI, oxígeno disuelto (OD) y dióxido de carbono $\left(\mathrm{CO}_{2}\right)$ mediante Kit de Aquamerck, saturación de oxígeno $(\% \mathrm{OD})$, Nitratos $\left(\mathrm{NO}_{3}^{-1}\right)$, carbonatos $\left(\mathrm{CO}_{3}^{-2}\right)$, dureza total y carbonacea, usando los "kits" de reactivos analíticos colorimétricos Aquamerck, $\mathrm{pH}$ con un $\mathrm{pH}$-metro (ShottGerate), la conductividad utilizando un conductímetro (YSI) y penetración lumínica mediante el disco de Secchi. La toma de muestras de agua a diferentes profundidades se realizó con un muestreador Kemmerer.

Determinación de clorofila a. Se determinó la concentración de pigmentos fotosintéticos presentes en distintas muestras de agua del humedal para lo cual se tomaron muestra de agua en dos zonas; la zona 1 (litoral) y la zona 2 (Linmética). Se determinó la zona fótica utilizando el disco Secchi, y se tomaron muestras a 1, 10 y $50 \%$ de la penetración lumínica, lo correspondió a 90, 50 y $10 \mathrm{~cm}$ de profundidad respectivamente. Se midieron las concentraciones de clorofila a, la cual permite estimar la biomasa de la mayor parte del fitoplancton. Esto se llevó a cabo mediante la utilización del método tricromático (JEFFREY y HUMPHREY, 1975) y el índice de estado trófico de CARLSON (1977). Aplicando la ecuación:

$\mathrm{IET}=10(6-2.04-0.68$ 1n [clor.a] $\div$ 1n2

Vegetación ribereña y litoral. La caracterización de la vegetación, se realizó a partir de una colecta libre por la franja de la madre vieja, las muestras fueron depositadas en bolsas plásticas, las cuales se prensaron en papel periódico y montaron tipo herbario para su posterior identificación. Se describieron las características morfológicas sobresalientes (formas y colores). Igualmente, se estudió la distribución de la vegetación de la zona del humedal (RANGEL y VELÁSQUEZ, 1997).

Macroinvertebrados acuáticos. La colecta de macroinvertebrados acuáticos epicontinentales asociados a las macrófitas flotantes presentes en el espejo de agua; se sometieron a procesos de lavado a nivel radicular, con el fin de obtener el mayor número de muestras, para su posterior identificación, y cálculo del índice biológico BMWP (Biological Monitoring Working Party Store System) propuesto por ARMITAGE et al. (1983), adaptado para Colombia por ZAMORA (1999, 2005 y 2007) y ROLDAN (2003). 


\section{Resultados y discusión}

El estudio batimétrico en una longitud total de $72 \mathrm{~m}$ en una de sus partes más anchas, presenta una profundidad media de $2,5 \mathrm{~m}$ y máxima de $3,3 \mathrm{~m}$, en la parte litoral con una profundidad mínima de $60 \mathrm{~cm}$ en época de alta pluviosidad y en época de baja pluviosidad la columna de agua presenta una profundidad media de $1,80 \mathrm{~m}$ y máxima de $2,10 \mathrm{~m}$, y en la parte litoral con una profundidad mínima de $20 \mathrm{~cm}$. Los parámetros físicos y químicos obtenidos se muestran en la Tabla 1.

Tabla 1. Parámetros físico- químicos de la Madre vieja.

\begin{tabular}{lc}
\multicolumn{1}{c}{ Parámetro } & Registro \\
\hline Temperatura hídrica $\left({ }^{\circ} \mathrm{C}\right)$ & 27 \\
Oxigeno disuelto $(\mathrm{mg} / \mathrm{L})$ & 3.0 \\
$\%$ de saturación & 40 \\
Dióxido de Carbono $\left(\mathrm{mgCaCO}_{3} / \mathrm{L}\right)$ & 10.3 \\
Dureza Total $\left(\mathrm{mgCaCO}_{3} / \mathrm{L}\right)$ & 67.9 \\
Dureza Carbonatos $\left(\mathrm{CaCO}_{3} / \mathrm{L}\right)$ & 37.4 \\
Nitratos $\left(\mathrm{NO}_{3}^{-}\right)-\mathrm{mg} / \mathrm{L}$ & 87 \\
Fosfatos $\left(\mathrm{PO}_{4}{ }^{-2}\right) \mathrm{mg} / \mathrm{L}$ & 5 \\
Conductividad $(\mu \mathrm{Mhos} / \mathrm{cm})$ & 350 \\
pH & 8.3 \\
\hline
\end{tabular}

El aporte excesivo de materia orgánica, por la utilización de fertilizantes en los cultivos agrícolas, y de materia inorgánica de origen alóctono, por escorrentía pluvial, los fuertes vientos y las intervenciones antrópicas, son razones por las cuales la alta tasa de descomposición orgánica y los procesos oxidativos hacen que las concentraciones de oxígeno disuelto encontradas sean bajas (3,0 mg/l), comparadas con las concentraciones de dióxido de carbono que son considerablemente altas (10,3 mg/l) (ROLDÁN, 1992). Teniendo en cuenta que estos dos parámetros son indicadores del estado trófico de cualquier sistema, se deduce que el sistema presenta un estado de eutroficación (ROLDÁN, 1992).

En cuanto al $\mathrm{pH}$, este tiene una relación inversa con los niveles de $\mathrm{CO}_{2}$, en esta madre vieja se encontró algo muy particular, con una concentración de $\mathrm{CO}_{2}$ tan alta se esperaba hallar un $\mathrm{pH}$ con tendencia ácida (ROLDAN, 1992) pero fue todo lo contrario, el pH registrado es básico $(8,3)$; la deducción de acuerdo con este parámetro, es la presencia de carbonatos que actúan "amortiguando" el sistema. Los registros de conductividad (350 $\mu \mathrm{Mhos} / \mathrm{cm})$ corroboran alta concentración de iones presentes en el humedal.

Esto causa una disminución notoria en los niveles de dióxido de carbono, gas requerido para que los productores lleven a cabo el proceso fotosintético, lo cual explica los valores de $\mathrm{CO}_{2}$ mínimos encontrados en todas las zonas de muestreo.

Al encontrar una alta tasa de actividad fotosintética que consume altas concentraciones de $\mathrm{CO}_{2}$, hace que en el medio presente altos índices de los iones orgánicos de carbonatos $\left(\mathrm{CO}_{3}{ }^{-}\right)$y bicarbonatos $\left(\mathrm{HCO}_{3}^{-}\right)$, que conllevan a la basificación del sistema (ROLDAN, 1992), corroborados por los registros del $\mathrm{pH}$ que oscilan entre 8,3 - 8,7 (Tabla 1).

El último aspecto a considerar es la conductividad que no presento variación para las diferentes zonas de muestreo siendo de $350 \mathrm{mMhos} / \mathrm{cm}$ (Tabla1), valor que está ligado a la dureza total (68 a $\left.85,4 \mathrm{mgCaCO}_{3} / \mathrm{L}\right)$, dada la abundancia de iones orgánicos de carbonatos y bicarbonatos, cuya clasificación desde el punto de vista de la productividad, según BOYD (1990), determina aguas duras en la orilla y muy duras hacia el centro del humedal, cuya evaluación comprueba que se trata de aguas muy productivas (eutróficas).

Determinación de clorofilas de la madre vieja Gota e' Leche. La cuantificación de la clorofila y específicamente la clorofila A, facilita elementos de juicio para determinar el estado actual y cambios dentro de un ecosistema acuático, lo cual representa un atributo ecológico significativo. Con base en los resultados expuestos, se detectan varios fenómenos relacionados con la capacidad trófica del humedal (Tablas 2 y 3 ).

Tabla 2. Determinación de clorofila A. Zona 1.

\begin{tabular}{|c|c|c|c|c|}
\hline Porcentaje luminosidad (\%) & Profundidad (m) & Determinación clorofila a (mg/L) & Determinación clorofila a $(\mathrm{mg} / \mathrm{m} 3)$ & Índice Estado Trófico \\
\hline 50 & 0.1 & 4.1064 & 410.64 & \multirow{4}{*}{108.5} \\
\hline 10 & 0.5 & 3.30 & 330 & \\
\hline 1 & 0.9 & 3.58 & 358 & \\
\hline \multicolumn{3}{|c|}{ Total } & 1098.64 & \\
\hline
\end{tabular}

Tabla 3. Determinación de clorofila A. Zona 2.

\begin{tabular}{ccccc}
\hline Porcentaje luminosidad (\%) & Profundidad $(\mathbf{m})$ & Determinación clorofila a (mg/L) & Determinación clorofila a $\left(\mathbf{m g} / \mathbf{m}^{3}\right)$ & Índice Estado Trófico \\
\hline 50 & 0.1 & 3.64 & 464 \\
10 & 0.5 & 4.50 & 450 \\
1 & 0.9 & 4.38 & 438 & 109.8 \\
\hline \multicolumn{7}{c}{ Total } & & 1252 \\
\hline
\end{tabular}


Los resultados indican que se trata de un sistema hipereutrófico para el Índice de Carlson (1977). Es un ecosistema que ha sufrido un alto grado de alteración causado por las actividades antrópicas adyacentes, y en donde se ha destruido casi en su totalidad la zona natural de amortiguación representada por la vegetación de esta zona de vida bs-T circundante. Adicionalmente se encuentran canales de irrigación que son aprovechados para el riego de la caña de azúcar, esta acción causa la incorporación directa de nutrientes, causando una descompensación en la capacidad de carga del humedal, mostrado por los valores encontrados en la determinación de la clorofila A y el índice de estado trófico.

Vegetación acuática y riparia. En la Figura 2, se observa la distribución de las plantas acuáticas en la madre vieja: flotantes, las familias más importantes y representativas son Pontederiaceae y Araceae; entre las comunidades marginales o enraizadas, se encuentran pastos, juncos, o las eneas o espadañas cuyos nombres científicos se indican en la tabla 4. En esta zona, la vegetación de ribera corresponde a un bosque donde dominan familias como: Salicaceae (sauces), Fabaceae (Cachimbos, chiminangos y zarzas) y Poaceae (pastos). Por último, hay que hacer mención a las profundas transformaciones que pueden apreciarse en la vegetación de un tramo de ribera. Dichas alteraciones tienen origen en el desarrollo de la sucesión ecológica, pero también a las variaciones geomorfológicas por intervención antrópica sobre la madre vieja.

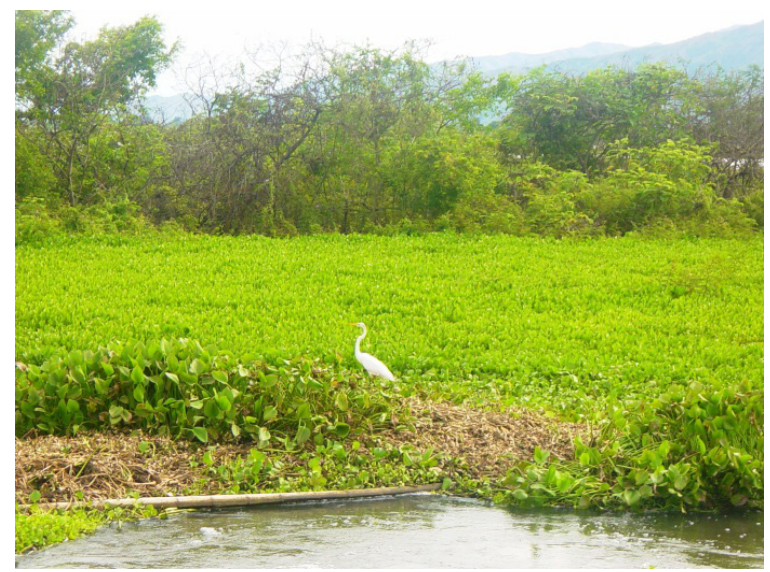

Figura 2. Vegetación acuática en la Madre vieja.

Fuente: Zambrano (2010)

Macroinvertebrados acuáticos epicontinentales MAE. Con los registros obtenidos a nivel de orden (Tabla 5). Se pretende conocer la calidad biológica del agua como un elemento más para el análisis del estado trófico del cuerpo de agua.
Tabla 4. Vegetación acuática y ribereña Madre vieja Gota e' Leche, municipio de Yotoco.

\begin{tabular}{|c|c|c|}
\hline Nombre común & Nombre científico & Familia \\
\hline Barbasco & Persicaria punctata (Elliott) Small & Polygonaceae \\
\hline Buchón de agua & Eichhornia crassipes (Mart.) Solms & Pontederiaceae \\
\hline Cachimbo & Erythrina fusca Lour. & Fabaceae \\
\hline Cañafístola & Cassia grandis L. f. & Fabaceae \\
\hline Ceiba & Ceiba pentandra (L.) Gaertn. & Malvaceae \\
\hline Chambimbe & Sapindus saponaria L. & Sapindaceae \\
\hline Chiminango & Pithecellobium dulce (Rob) Benth & Fabaceae \\
\hline Cordoncillo & Piper sp & Piperaceae \\
\hline Enea & Typha latifolia L. & Typhaceae \\
\hline Guadua & Guadua angustifolia Kunth & Poaceae \\
\hline Guácimo & Guazuma ulmifolia Lam. & Malvaceae \\
\hline Higuerilla & Ricinus communis $\mathrm{L}$. & Euphorbiaceae \\
\hline Junco & Eleocharis elegans (Kunth) Roem. \& Schult. & Cyperaceae \\
\hline Lechuguilla & Pistia stratiotes L. & Araceae \\
\hline Loto & Nymphaea sp & Nymphaeaceae \\
\hline Manteco & Laetia americana L. & Salicaceae \\
\hline Pasto & Hymenachne amplexicaulis (Rudge) Nees & Poaceae \\
\hline Samán & Samanea saman (Jacq.) Merr. & Fabaceae \\
\hline Sauce & Salix humboldtiana Willd. & Salicaceae \\
\hline Yarumo & Cecropia sp & Urticaceae \\
\hline Zarza & Mimosa pigra L. & Fabaceae \\
\hline
\end{tabular}

Tabla 5. Valores del BMWP para MAE encontrados en la madrevieja.

\begin{tabular}{cccc}
\hline Clase & Orden & Familia & Puntaje BMWP \\
\hline Insecta & Odonata / Anisoptera & Libellulidae & $(6)$ \\
Insecta & Odonata / zigoptera & Coenagrionidae & $(9)$. \\
Insecta & Ephemeroptera & Baetidae & $(8)$ \\
Insecta & Coleoptera & Noteridae & $(4)$ \\
Insecta & Coleoptera & Elmidae & $(7)$ \\
Insecta & Coleoptera & Dysticidae & $(8)$ \\
Insecta & Coleoptera & Hydrophilidae & $(4)$ \\
Insecta & Diptera & Chironomidae & $(2)$ \\
Insecta & Diptera & Culicidae & $(3)$ \\
Insecta & Diptera & Simuliidae & $(9)$ \\
Insecta & Hemiptera & Naucoridae & $(7)$ \\
Gastropoda & Architaenioglosa & Ampullaridae & $(9)$ \\
Gastropoda & Bassomatofora & Physidae & $(3)$ \\
\hline & Total, sumatoria BMWP & & 79 \\
\hline
\end{tabular}


Tabla 6. Clases, Valores y Características para aguas naturales clasificadas mediante el índice BMWP. Fuente: (ZAMORA, 2007).

\begin{tabular}{ccccc}
\hline Clase & Rango & Calidad & Características \\
I & ${ }^{3} 121$ & Muy Buena & Aguas muy limpias & Color Cartográfico \\
II & $101-120$ & Buena & Aguas limpias & Azul claro \\
III & $61-100$ & Aceptable & Aguas medianamente contaminadas \\
IV & $36-60$ & Dudosa & Aguas contaminadas & Amarillo \\
V & $16-35$ & Crítica & Aguas muy contaminadas \\
VI & $£ 15$ & Muy crítica & Aguas fuertemente contaminadas
\end{tabular}

Los organismos de la muestra capturada fueron identificados taxonómicamente a nivel de familia, y con base en los resultados obtenidos se asignó el puntaje correspondiente a cada familia de acuerdo a la comparación con el BMWP adaptado para Colombia por (ZAMORA, 2007, 2005; ROLDÁN, 2003). El valor de la sumatoria fue de 79, lo cual significa que los MAE de la zona de estudio presentan características de agua de calidad medianamente contaminadas.

En el caso de la clase Gastropoda se encuentra que los géneros Pomacea y Physa, encontrados en grandes proporciones poseen elevada capacidad de sobrevivir en aguas contaminadas y/o muy contaminadas. Para el orden Coleoptera, especialmente las familias Dytiscidae e Hidrophilidae, es común encontrarlas en sistemas lénticos con valores de temperatura, conductividad y de dióxido de carbono por encima de lo normal, es decir, con moderado grado de contaminación. Esto sumado a la presencia del orden Díptera especialmente la familia Chironomidae, que son indicadores de aguas medianamente eutroficadas (ROLDAN, 1996).

En conclusión, se trata de un ecosistema hiper eutroficado, con calidad de aguas caracterizadas de mediana contaminación que ha sido severamente afectado por las actividades antrópicas. Dada que el humedal se encuentra en un área dedicada al cultivo de la caña y sometido intensamente a las prácticas relacionadas con este cultivo será difícil recuperar las características naturales del sistema, a no ser que la autoridad ambiental lo catalogue como un humedal a proteger y cese su uso antrópico.

\section{Referencias}

AMERICAN PUBLIC HEALTHASSOCIATION. 1999. Standard methods for the determination of water and wastewater. $20^{\text {th }}$ Edition. APHA, New York, USA.

ARMITAGE, P.D.; MOSS, D.; WRIGHT, J.F.; FURSE, M.T. 1983. The performance of a new biological water quality score based on macroinvertebrates over a wide range of unpolluted running-water sites. Wat. Res17:333-347.

BOYD, C.E. 1990. Water Quality in ponds for aquaculture. Alabama Agricultural Experiment Station. Editorial. Auburn. AL, USA.

CARPENTER, S.R.; COTTINGHAM, K.L. 1998. Resilience and Restoration of Lakes. Conservation Ecology.

BARBOUR, M.T., J. GERRITSEN, B.D. SNYDER, AND J.B. STRIBLING. 1999. Rapid Bioassessment Protocols for Use in Streams and Wadeable Rivers: Periphyton, Benthic Macroinvertebrates and Fish. Second Edition. EPA 841-B-99-002. U.S. Environmental Protection Agency. Office of Water. Washington, USA.

GUERRERO, E. 1998. Una aproximación a los humedales en Colombia. 1. Ed. Colombia: FEN. Bogotá, Colombia.

HOLDRIGE, L.R. 1978. Ecología basada en zonas de vida. Instituto Interamericano de Cooperación para la Agricultura (IICA). Serie de libros y materiales educativos No. 34. San José, Costa, Rica.

JEFFREY S.W.; HUMPHREY, G.F. 1975. New spectrophotometric equation for determining chlorophyll a, b, c1 and c2, Biochem. Physiol. Pflanz. 167:191-194 
RANGEL-CH, J.O.; VELÁSQUEZ, A. 1997. Métodos de estudio de la vegetación. Págs. 58-87. En: Rangel, J.O.; Lowy-C, P.; Aguilar, P. M. (ed). Colombia Diversidad Biótica II. Santafé de Bogotá: Instituto de Ciencias Naturales. Universidad Nacional de Colombia. Colombia.

ROLDAN, G. 1992. Fundamentos de limnología neotropical. Editorial Universidad de Antioquia. Medellín, Colombia.

ROLDÁN, G. 1996. Guía para el estudio de macroinvertebrados en el departamento de Antioquia. Universidad de Antioquia. Medellín, Colombia.

ROLDÁN, G. 2003. Bioindicación de la calidad del agua en Colombia. Uso del método BMWP/Col. Editorial Universidad de Antioquia. Medellín. Colombia.

WAYNE-NELSON, R.; WELLER, E.C. 1984. A better rationale for wetland management. Environmental Management 8 (4):295-308.

ZAMORA, H. 1999. Adaptación del índice BMWP para la evaluación biológica de la calidad de las aguas epicontinentales en Colombia. Revista Unicauca-Ciencia 4:47-60.

ZAMORA, H. 2007. El Índice BMWP y la Evaluación Biológica de la calidad del Agua en los Ecosistemas Acuáticos Epicontinentales Naturales de Colombia. Revista de la Asociación Colombiana de Ciencias Biológicas 19:73 - 81. 